International Journal of Engineering \&Technology, $7(1.8)(2018)$ 182-187
International Journal of Engineering \& Technology
SPC
Website: www.sciencepubco.com/index.php/IJET
Research paper

\title{
A Review on Current Methods of De-Icing and an Idea for Designing an Autonomous Robot for De-Icing
}

\author{
Dr. P. Srinivas Verma ${ }^{1 *}$, Batchu Sidharth Sarat Raj ${ }^{2}$, Sandopu Sainath ${ }^{3}$, Eyerrmshetty Kailash Narsingh Rao ${ }^{4}$ \\ ${ }^{1}$ Assoc. Professor, Department of Electrical and Electronics Engineering, Koneru Lakshmaiah Education Foundation, Guntur, India \\ ${ }^{2,3,4}$ UG Student, Department of Electrical and Electronics Engineering, Koneru Lakshmaiah Education Foundation, Guntur, India \\ *Corresponding author E-mail: pinniverma@ gmail.com
}

\begin{abstract}
Extensive need regarding research and development of new techniques regarding transmission conductor de-icing is observed with the current methods available, are highly unsustainable to rely on as they are risky not only to the power system stability but also the lives of people and operations in the load area. This paper holds a technique and an idea for ice removal mechanism of transmission line that could be easily implemented, mass produced and works autonomously along with review on current methods. A robot for every span of transmission line which is self-sustained and completely autonomous to de-ice the transmission line with extremely cost efficient is objective being for this paper. Prototype model, working for dummy transmission system (without ice) shows good results for being applied to an original transmission line. De-icing here is obtained by applying constant friction on the transmission conductor which apparently does not cause damage to health and wear of the conductor. All the considerable measurements for that span of electrical system are tired to be met while designing. The performance approach is evaluated through practical simulation of a likely prototype which is subjected to be real.
\end{abstract}

Keywords: De-Icing; Transmission line; Robot; Autonomous; Prototype.

\section{Introduction}

A peculiar but not significantly looked problem is icing of transmission lines. Icing up of transmission line is a common natural disaster in places like Quebec in Canada, South Western America, Russia, Japan, Britain, Finland and Iceland caused security incidents because of getting coated with Ice on the transmission line. Ice formation on the transmission line causes stability issues of the power grid as lines tend either to break or come inside Ground Clearance areas. There are basically three types of transmission line De- icing in practice. They are mechanical deicing, passive method and mechanical De-icing. All the current techniques have not only removal of load for the duration of transmission line deicing but also are energy thirsty methods. Implementing a robot appears to be an ideal solution for this problem by looking at the robot developed by the Hydropower Research Institute in Quebec, Canada, which was a remotely operated and highly impractical to use out of labs not only because of cost but also inability to De-ice efficiently. Because of the reason robots are not only advantages in energy saving but in flexibility, and also in adaptability, Deicing robot is increasingly becoming a focus for this problem of mechanical deicing on transmission lines. Researchers at Quebec Hydropower Institute "Expliner" electric robot which is a Line Rover, seemed to be early as a manifest of deicing robot; it is mainly used to remove the ice of the transmission line. In 2006, this improved robot of transmission line also has the function of overcoming the barrier for transmission line De-Icing. The deicing robot with multiarmed is more and more effective and best variety which is developed and improved. However, the main disadvantage in installing the robot and conditioning the robot when malfunction occurs. Early method was using of heads with a blade scraping and knock which was a highly unstable and inefficient in the span of wire. These blades scraping and knock are more to work as damagers of the line rather that Ice remover after considering the above problem, this paper proposes a local cheap method for deicing method, which can be implemented by the head of the deicing robot to produce incentive displacement to vibrate the transmission line particularly at each point due to which ice break off from the wire, so as to meet the objective of the prevention of the transmission line. In this technique this vibration is caused by a constant and continues running motor with unsymmetrical load due to which it acts as a vibrator. As in the early stages of ice formation of transmission line, Ice is extremely brittle that it does not require hard scraping, just a simple mocking or vibration motion could remove the ice. Transmission line distance between two transmission towers is called span. To reduce the tension of the line and to allow free play during windy conditions some amount of sag is introduced in the transmission line. Sag is amount of value of distance from the point where transmission line is connected to the transmission tower to the lowest point of the line. Sag of the transmission line must not be either too low or too high. It must be within safety limits. If sag is not introduced, then in winter the metal conductor contract and line break due to which a well-engineered safely limited sag is introduced. Sag should never go below the ground clearance level. Icing of transmission line is extremely dangerous.

$$
\begin{aligned}
& \operatorname{Sag}(S)=\frac{w_{l}\left(\frac{l}{2}\right)^{2}}{2 T} \\
& =\frac{w_{l} l^{2}}{8 T}
\end{aligned}
$$


Where $\mathrm{w}$ is the weight of the conductor,

$\mathrm{L}=$ length of the line.

$\mathrm{T}=$ tension

$\mathrm{W}_{\mathrm{l}}=$ weight of the conductor for unit length 1 .

Let us assume weight of conductor without ice $=\mathrm{W}$.

Weight of conductor with ice $=\mathrm{W}_{\mathrm{i}}$

Then,

$$
w_{t}=\sqrt{\left(w_{l}+w_{i}\right)^{2}+\left(w_{w}\right)^{2}}
$$

$\mathrm{W}_{1}=$ weight of the conductor per unit length 1

$\mathrm{w}_{\mathrm{i}}=$ weight of ice per unit length

$$
\begin{aligned}
& w_{i}=\text { Density } \times \frac{\Pi}{4}\left[(d+2 t)^{2}-d^{2}\right] \times l \\
& w_{i}=\text { Density } \times \Pi t(d+t)^{2}
\end{aligned}
$$

$\mathrm{W}_{\mathrm{w}}=$ Wind force per unit length

$=$ Wind pressure $\times[(d+2 t) \times 1]$

$\mathrm{w}_{\mathrm{t}}=$ total weight of the conductor

From the above equation it is evident that increase in ice on the transmission line increases the weight of conductor due to which the sag increases due to which transmission line comes into ground clearance area and if more amount of ice accumulates and once it goes beyond the maximum bearable tension limit, then the transmission line conduction stops, Which causes line to be replaced and thus drives the entire grid into a dangerous and unreliable system in cold winters when the conductors are iced. Thus, an imperative maintenance during winter is extensively required.

\section{Review of Current Techniques}

\subsection{Mechanical De-Icing:}

This method involves using of external mechanical forces to deice or break the ice on transmission lines. By using a pry or a rod, the ice is being knocked off the transmission line manually. This needs and assignment of workers in shifts. The other method that comes under this category is by inserting a pulley and pulling it throughout the line to clean the ice off the line.

\section{Disadvantages involved:}

This process needs additional equipment to clean the ice off the lines. And involves manual effort, and it is highly time consuming. So, shifts must be taken in order to monitor the line continuously. This method may be less safe under extreme weather conditions especially in cold countries. Knocking off the ice from the lines need to shut down the transmission line or supply for long durations to prevent swinging of lines and short circuits. This may lead to long interruptions or outages. This method is highly prone to accidents or human induced faults while de-icing.

\subsection{Thermal De-Icing:}

This method involves developing heat in the transmission line that needs to be de-iced. The heat generation process in this method is often called as joule effect. This can be achieved by passing huge amounts of current through the transmission line and generating enough heat to melt off the ice. This makes the transmission line glow like a filament in the bulb due to resistance to such huge current. This method is also called short circuit de-icing. It is the most common method of de-icing.

Advantages of using this method:
Since most of the transmission lines used now a day's use A.C supply from grid, short circuiting can be made easier. This method is much time efficient when compared to other mechanical methods. In fact, it is the most commonly used method these days. Using short circuit method, instead of using auxiliary D.C supply, can use A.C supply directly from grid.

Disadvantages involved:

This method obviously needs to be implemented only when the transmission line is off the grid or supply to load because it cannot send high currents when the line is connected to the grid. So, this causes outage. The large currents are produced either by installing an external or auxiliary source or by increasing the burden of load on a single desired transmission line. To generate enough current and enough heat, the voltage across the transmission line may exceed the rated values or limits. So, the extent to which it can use this method is limited highly. This method is expensive compared to mechanical methods. Though it may not best time consuming as the mechanical de-icing methods. Another way to generate enough heat is by intentionally short circuiting the line. So, additionally care to be taken to make sure relays at the operational zones are not operating to draw power. (Because, the supply from generating station acts as the source that feeds the short circuit fault).There is a need for considering mechanical limits and thermal limits of the transmission line while using this method. So, the flexibility is limited. However, if the current exceeds thermal limit, two lines or conductors could be connected in series so as to increase voltage and reduce current to a limited value.

\subsection{D.C de-icing in A.C lines:}

This method also comes under thermal de-icing of the transmission line however the only difference is that, usage of external D.C supply to generate heat in the line is required. However, the most preferred method is using rectifiers and taking the A.C. supply directly from grid and converting into D.C. To adjust de-icing voltage, there is a need to use rectifier transformer and SCR (silicon-controlled rectifiers) in this method.

\section{Advantage of using this method:}

It increases flexibility by giving us chance to adjust the de-icing voltage across line to desired limit so that enough current is generated in the line with in thermal limits.

\section{Disadvantages involved:}

This method clearly needs more equipment that needs to be installed than the one involving short circuiting the line. So, it is very costly and less preferred as there are maintenance issues of the additional equipment also. Additional protection equipment must also be implemented for the rectifier transformer and SCR units. This method can use only one line at a time. So, there is no flexibility of connection of other line in series to limit current in the required line with in limit. Supply to loads needs to be cut off while using this method in the transmission line.

\subsection{Mobile D.C. Power Supply De-Icing:}

This method involves a mobile (auxiliary) D.C supply that needs to be moved along the length of the transmission line to power the current that generates heat in the line.

Advantages of using this method:

The equipment required is less compared to previous method that taps power from line itself. The amount of de-icing significantly depends on length of the transmission line but not on the supply.

\section{Disadvantages involved:}

This method needs additional power supply, it is expensive. Requires more manual effort and is time consuming. The maximum mobile dc power supply is limited. 


\subsection{Reactive Power De-Icing:}

In this method, reactive power is supplied or injected into the transmission line to increase the current to generate heat. This also comes under thermal de-icing. In this method, valve is to be adjusted for inductor reactance which is at load side to change the amount of current in flowing in the conductor, while shunt capacitor is placed at power system side

Advantages of using this method:

Installation of capacitor that are used for shunt compensation and parallel adjustable inductor are effortless with small amounts of workload.

\section{Disadvantages involved:}

Installation of additional equipment like shunt capacitor and inductor bank is required. It is especially difficult to control reactive power while the line is in loop of the network. Adjusting reactive power may cause unsteady problems to the power system. By injecting additional reactive power into the line, power factor adjustment may be a problem. This method cannot be used when the transmission line is under operation.

\subsection{Phase shifting transformer De-Icing:}

In this method, a phase shifting transformer is used. Phase shift transformer could create an active power circle in which one line transmits forward while the other line transmits reverse. So, this forward transmission current in addition with phase alternating current along with load current which creates circulating current to flowing in a transmission line and these currents causes the effect of de-icing. During the ordinary operating conditions in this process the ice of the conductor line is melted by tuning the circulating current as the double circuit transmission conductors.

Advantages of using this method:

This method is used while operation of the transmission line. This doesn't cause any interruption to supply. So, it is most popular these days.

\section{Disadvantages involved:}

Phase-shifting transformer is additional to the main circuitry adding more components to the circuit excluding the protection elements for it, and consumes more reactive power, which can influence the steadiness of system.

\subsection{Using Dielectric Losses to De-Ice Power Transmission Lines With 100 kHz High- Voltage Excitation:}

In this method, an auxiliary power supply of about $100 \mathrm{kHz}$ and high frequency and about $30 \mathrm{kv}$ power source is connected to the transmission line. The ice layer in between the ground and transmission line has some value of resistance and capacitance that adds up to capacitance of transmission line w.r.t. ground. For small frequencies lesser than $8 \mathrm{kHz}$, then dielectric properties become resistive capable to generate enough heat to melt off the ice from transmission line. If supplied frequency is increased, the voltage needs to create sufficient loss. Generally opted range for operation is near $100 \mathrm{kHz}$. At a frequency between 60 to $100 \mathrm{kHz}$, on a $33 \mathrm{kV}$ is sufficient to produce a heating of $50 \mathrm{~W} / \mathrm{m}$ on a $1 \mathrm{~cm}$ sheath of ice.

\section{Advantages of using this method:}

High frequency degrades dielectric properties of ice and generates heat to melt off ice. So, as this can be achieved without damaging thermal properties of conductor or line it is more preferred to DC source de-icing.

\section{Disadvantages involved:}

All this energy is to be processed by any power source at one end while absorbed after the termination at the other end. Making this noticeable, the capability of the source must be increased much beyond the power required for heating. This termination must be capable of dissipating, bearing or recycling this power. Making this process an expensive solution. There is a need to isolate the transmission line from supply or grid. So, this is not desired. To generate high frequency supply $(60-100 \mathrm{kHz})$, additional equipment near source i.e. power source inverter is required.

\subsection{Remotely Operated Vehicle:}

In this method a mechanical robot for ice removal on the overhead wires in the grid is developed. This method allows slow de-icing of transmission conductors, without creating sudden stress on structures, being the main objective. The device has high traction force robust, lightweight, and compact, which allows it to perform the task of removing ice of the transmission lines. The ROV was successfully tested on live conductors $(315 \mathrm{kV})$ while having its circuitry protected from the electromagnetic interference.

\section{Advantages of using this method:}

The main advantage in this method is that there is scope of removing transmission line ice with minimum requirement of maintenance and apparently minimum number of operators required. There is no need to turn off the supply through the transmission line while operating the robot, thus preventing power interruption which is an important requirement. It is time efficient and requires almost no manual effort and very less chances of failures or dangers. There's no need not disturb any relay operation in any zone of transmission line.

\subsection{High - Voltage Short - Circuit Method:}

The effect which causes heating of a conductor when current passes through it if Joule effect, for applying it is applied to a transmission line, it should be short circuited which could not be applied usually to bundled conductors (as bundled conductors are designed to overcome Joule effect). So, a technique which is developed for involving short circuit currents $\left(\mathrm{I}_{\mathrm{sc}}\right)$ at rated voltage and rated frequency and nominal working conditions of transmission network which causes a sufficient and succeeding response of electromagnetic forces that make transmission line conductors to flick each other and make ice break off the line.

Advantages of using this method:

This method can significantly remove most of the ice in one-time application of the method.

\section{Disadvantages involved:}

This method causes increases in the wear of the line as transmission line is tended to electromagnetic force which causes the transmission line to move rigorously causing the joints to loosen and eventually increases the sag and results in corona at the junctions.

\section{Proposed Methodology for Robotic De - Ic- ing:}

All the working of the robot is classified into parts (sub blocks) to create an ease in the implementation and development of code for the microcontroller and also implementation of the additional features in the future if implemented in future.

\section{Distance Monitoring System:}

Every transmission line span will have two ends. Each one being bounded by an insulator string. 


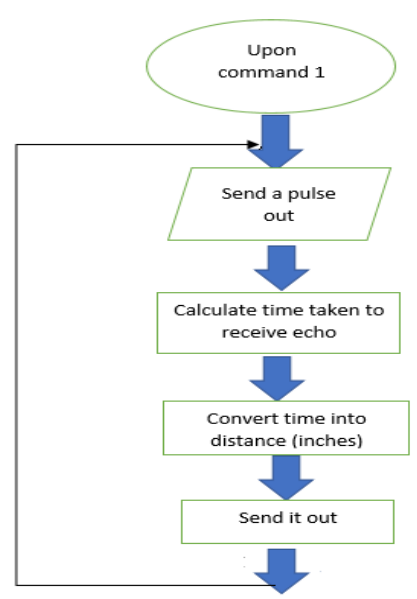

Fig: 1.2 Distance monitoring system block diagram.

So, the methodology should include a pair of sensors looking for obstacles both in front and back of the robot. Out of many, ultrasonic and IR have got the attention and IR sensor is ruled out because of not having sufficient strength to detect object such as insulator string. So, only an ultrasonic sensor would serve. The obstacle sensor must follow the algorithm (fig 1.2). First, the ultrasonic sensor sends out a signal that reflects from obstacles (like insulator strings) and is received by the receiver an echo signal. The time delay taken to reach is calculated in the processor. And it is further converted into distance (in inches) then the signal is further sent to motors based on pre-set conditions in the code. I.e. when the insulator string is nearer than a pre-set value the signal is sent to servo motors.

Thus, distance is to be measured with the help of receiving and transmitting pulses. The pulses are sent to the controller board where it checks for the analogy and then decides wither to go forward or to stop.

Two of these distance monitoring systems are to be embedded to detect forward insulator string distance and backward insulator string distance. This system should detect distance and send both the distances to controller board. The controller board then issues a signal to motor control unit directly which sends signal to servo motors to move in a direction.

\section{Temperature Monitoring System:}

LM35D3 is a high quality with controlled output, integrated circuit temperature sensor where output voltage is proportional linearly to the scale of Celsius and it being a low power consuming device draws only 60 micro amperes from the supply. Being able to operate in between -55degree Celsius to 150 degrees Celsius, makes this sensor ideal for this purpose.

Flow chart of temperature monitoring system:

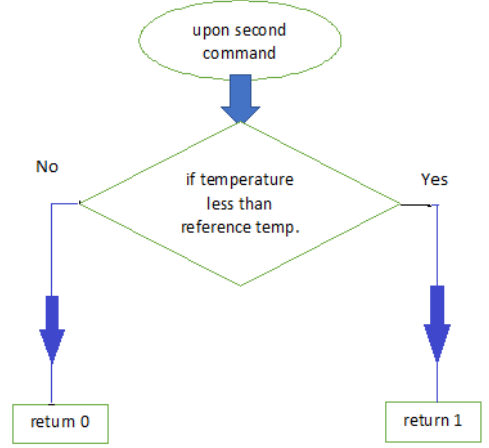

Fig 1.3 Block Diagram For Temperature Monitoring System

\section{Motor Driver Circuit:}

The main source of this application being transmission line deicing, it requires a humongous torque, but speed is not concerned making us to use a low cost high torque DC motor which is reversible. A motor driver with small value of voltage and small value of power consumption is desired there by making L293D IC an opt one.

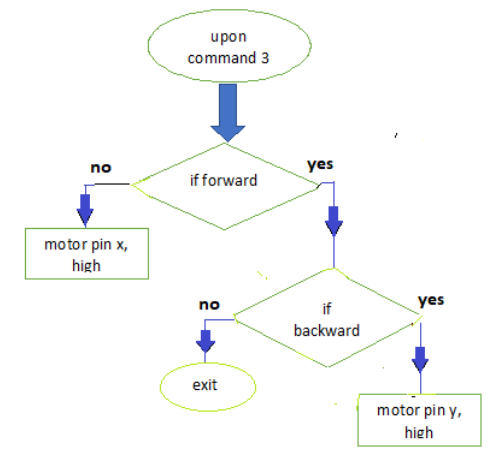

Fig:1.4: Block Diagram For Motor Controller

\section{Implementation or Aggregation:}

All the above developed methods are to be carefully implemented and aggregated with tuning the values of sensor and custom fitting the reference values This robotic system being completely autonomous, must keep sensing temp. Transmission conductor, once the temperature drops below the reference, here being 0 degrees Celsius, the robot should decide to go forward or backward depending upon the obstacle present in its course. Obstacle being the insulator string. Once the direction is decided the controller sends a command to motor controller IC to roll towards the direction decided until another insulator string is sensed and then the same process is repeated.

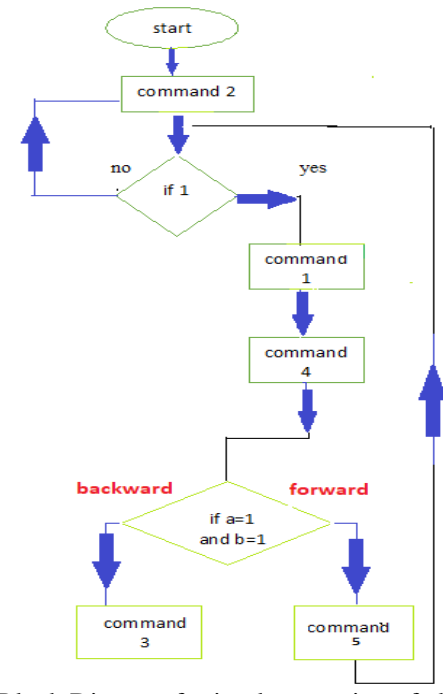

Fig:1.5: Block Diagram for implementation of all the units

\section{Developed Prototype:}

Utilizing all the above blocks, a multi-arm robot has been developed keeping in the view of electromagnetic interference and practical heights and sizes. This prototype has significant drawbacks regarding mechanical model and framework. Limited knowledge regarding the mechanical designing of structure has lead failure of the first model. The second prototype is made with complete idea and different theory and also considering the mechanical limitation some of the capabilities of this robot are meaningfully and intentionally reduced 


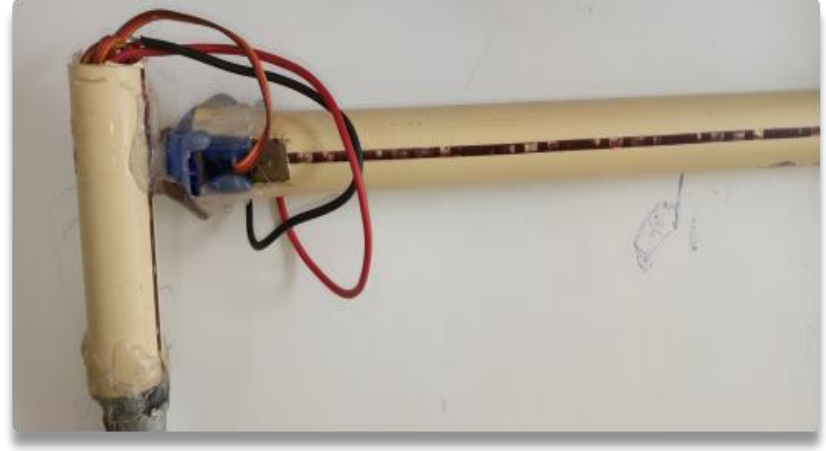

Fig:1.6 Showing the failed model cantilever beam and Rotary arm.

Concept of Second Prototype:

Considering the limitations, a second prototype is made with the ideology of using one robot for one span so that the amount of burden on each robot is not only reduced but also there is an easefor the robot to operate with reduced burden

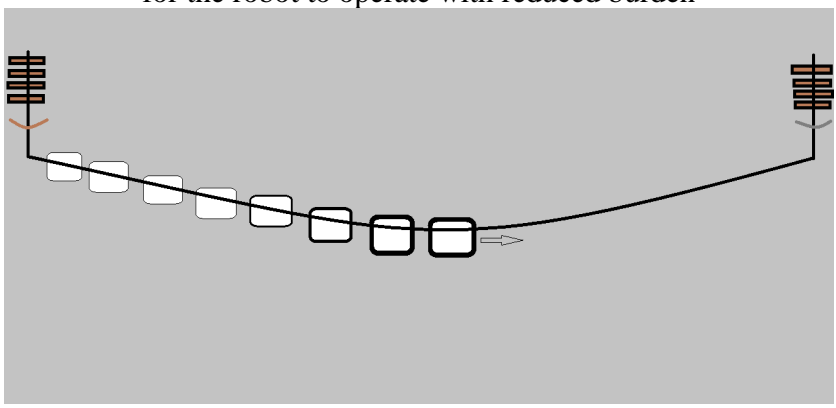

Fig:1.6: Second Model Working Idea.

and de-icing would become anti-icing i.e. this robot starts its operation quite earlier than the previous one so as by grappling motion on the transmission line.

Imaginary Model of Second Prototype:

This imaginary model has all same working and constructional details as the earlier one except those facts

- It would be acting as Anti-Icing instead of De-Icing.

- One robot should be placed for every span of transmission line.

- Much cheaper compared to previous model.

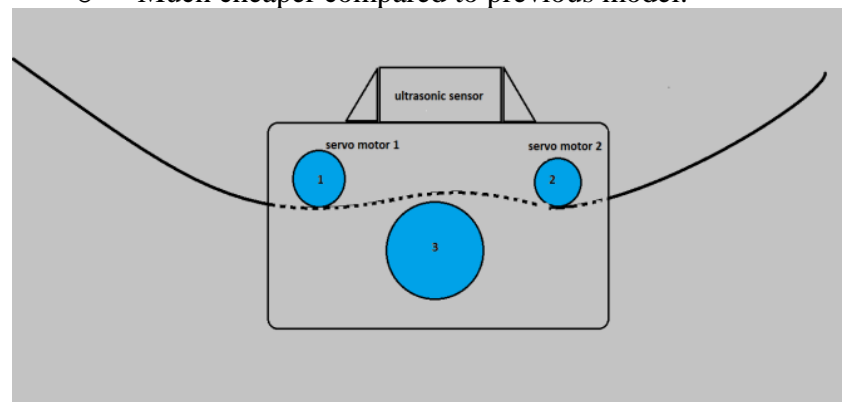

Fig:1.7:Model Diagram of Second Prototype

Much lesser maintenance compared to previous model.

More efficient as it is dealing with Anti-Icing.

Lesser weight when compared to previous.

Less moving components.

Difficulty to attach or detach.

Difficulty in installing when compared to previous.

Thus, by making the second model more acceptable and convenient to make.

\section{Future Implementations:}

This model can be connected through a local wireless connectivity and transmission line inspection would be implemented. If gone further, this robotic system could be upgraded into a fault isolation and as basic repair implementation robot. By incorporating some nature inspired ideas by which model one could cross the insulator string perfectly without fail and thereby reducing the required number of Robots and may be this robot could be extended to use for an entire stretch of transmission conductors. Insulator string cameras can be embedded and theft monitoring and fault inspection as well as implementation of smart controlling system would make the robot completely autonomous.

\section{Conclusion:}

These Models are designed and developed so as to produce more effective way to removal of ice formed on the transmission line. two models being developed but due to limitations of the first model the idea regarding first model is discarded. While second one could be developed to yield a successful developed and working and fully automated robot.

\section{References}

[1] G. Eason, B. Noble, and I.N. Sneddon, "On certain integrals of Lipschitz-Hankel type involving products of Bessel functions," Phil. Trans. Roy. Soc. London, vol. A247, pp. 529-551, April 1955. (references)

[2] Effect of Sag on Transmission Line Oluwajobi F. I., Ale O. S. and Ariyanninuola A. Department of Electrical and Electronics Engineering Technology Rufus Giwa Polytechnic Owo, Ondo State, Nigeria.

[3] Development of a Robotic Arm for Dangerous (Explode) Object Disposal

[4] 1 J.Dhanasekar,2P.Sengottuvel, 3 G.Manikandan, 4M.Deepa Assistant Professor, Department of Mechatronics, Bharath University, Chennai, India Professor, Department of Mechatronics, Bharath University, Chennai, India

[5] " The Effect of Changing the Conductors Layout on the Magnetic Field Level Generated Around Conductors of 132kv High Tension Transmission Lines Extended In Kirkuk City" Maryam Mohammed Falih

[6] "Preventing Transmission Line Damage Caused by Ice with Smart On-line Conductor Monitoring "by Nenad Gubeljak

[7] "Overhead Transmission Lines Deicing under Different Incentive Displacement" by Qing He, Xi-feng Lv, and Xiao-tong Zhao

[8] "Ultrasonic Sensor System for Ecology Observation and Timing Synchronization of ZigBee Network" by Atsushi MORIKAWA, Hiroshi YAMAMOTO, Maki YAMAMOTO, Katsuyuki YAMAZAKI

[9] "A Review Paper on Icing and Methods to De-Ice the Transmission Line" by Deepika Sinha, Srijana Bharadwaj, Asst. Prof. Vikas Kumar

[10] "Wind and environmental effects on overhead high voltage transmission lines" by A. F. Abdel-Gawad1, \& A.-S. A. Zoklot

[11] "Calculations of maximum sag of a transmission line with an ice load on one span" by Rodney A. Schaefer

[12] "De-icing EHV Overhead Transmission Lines by Short-circuit Currents" by Michel Landry, Roger Beauchemin and André Venne Hydro-Québec, Varennes, QC

[13] "Breaking the ice: de-icing power transmission lines with highfrequency, high-voltage excitation" by Sullivan, C. R. Petrenko, V. F. McCurdy, J. D. V. Kozliouk From IEEE Industry Applications Magazine, vol. 9, no. 5, pp. 49-54.

[14] "Android Controlled RC Car Unit" by Sujata Rathod, Vinay Bansal Computer Department Smt Indira Gandhi College of Engineering Navi Mumbai, India and Kishor T. Patil Computer Department Smt Indira Gandhi College of Engineering Navi Mumbai, India IJITE Vol.04 Issue-04, (April, 2016) ISSN: 2321-1776 
[15] "Determination of Current Required to De-ice Transmission Line Conductors" by Pascal Prud'Homme, Marc-Olivier Roux, HydroQuébec TransÉnergie, Pierre Guilbault, Paul-Antoine Séguin, Hydro-Québec Research Institute,Éric Hounkpatin, Hydro-Québec Équipement

[16] "Visionbased systems for autonomous driving and mobile robots navigation Ubiquitous computing seminar - FS2014" by Lukas H“afliger

[17] "A Real-Time Method to Detect and Track Moving Objects (DATMO) from Unmanned Aerial Vehicles (UAVs) Using a Single Camera" by Gonzalo R. Rodr'iguez-Canosa , Stephen Thomas , Jaime del Cerro , Antonio Barrientos and Bruce MacDonald

[18] "The System Reliability Analysis of 1000 kV UHV Transmission Tower Based on Moment Method" by Maohua L, Zhengliang L, Dengke Yu

[19] "De-icing/Anti-icing Techniques for Power Lines: Current Methods and Future Direction" by C.Volat M. Farzaneh and A. Leblond

[20] "Analysis of Dicing Techniques and Methods of Overhead

[21] Transmission Line" by Xifeng LV, Qing HE 